\title{
Capability Assessment of Inkjet Printing for Reliable RFID Applications
}

\author{
Jussi Putaala, Juha Niittynen, Jari Hannu, Sami Myllymäki, Esa Kunnari, Matti Mäntysalo, Member, \\ IEEE, Juha Hagberg, Heli Jantunen, Member, IEEE
}

\begin{abstract}
In this paper, inkjet-printed silver traces and interconnections produced with the print-on-slope technique were used in an RFID structure operating in the UHF range. Underfill material was used to attach silicon RFID chips onto flexible, 125$\mu \mathrm{m}$-thick polymer substrates. The cured underfill was also used as a sloped surface for printing interconnection traces from the chip to the plastic substrate's radiators. Inkjet printing was performed in one phase, producing both the interconnections to the chip and the radiators. This enables the use of a single-phase continuous roll-to-roll compatible process instead of the commonly used two-phase stop-and-go process. To further investigate the behavior of printed low-temperature nanoparticle ink and its compatibility with different substrate materials, basic conductive traces were printed onto the substrates.

Thereafter, the structures were exposed to thermal/humidity tests at $85{ }^{\circ} \mathrm{C}$ temperature/85\% relative humidity ("85/85") for up to a 2000-hour period. To gain an understanding of the response of the structures under stressing, the samples were intermittently characterized using a read range measurement device, followed by removal of failed samples from the test. The samples were characterized also by optical imaging and fieldemission scanning electron microscopy (FE-SEM). The bulk conductive traces were characterized electrically by measuring their resistances during test breaks.

The results point out that, although some challenges are still to be overcome, inkjet printing is a feasible way of producing conductive traces for RFID structures, and that the print-on-slope technique is utilizable also in practical applications as a costeffective method with adequate reliability for producing interconnections between chip and substrate.
\end{abstract}

Index Terms-85/85 test, Direct chip attachment, Inkjet, Interconnections, Reliability

Manuscript received June 27, 2016. This work was supported by Meadville Aspocomp, Nokia, Premix, UPM-Raflatac, and the Finnish Funding Agency for Innovation (Tekes). J. Putaala acknowledges the support in the form of grants from the Finnish Foundation for Technology Promotion (TES), the Riitta and Jorma J. Takanen foundation, and The Nokia Foundation. M. Mäntysalo is supported by Academy of Finland grant No. 288945 and 294119.

J. Putaala, J. Hannu, S. Myllymäki, Juha Hagberg, and Heli Jantunen are with the Microelectronics Research Unit, University of Oulu, P.O. Box 4500, FI 90014 Oulu, Finland. Phone: +358-294-487-962; fax: +358-8-553-2728; e-mail: jussi.putaala@ee.oulu.fi (J. Putaala).

J. Niittynen is with the Meyer Burger B.V., Luchthavenweg 105657 EB Eindhoven, The Netherlands.

E. Kunnari is with the Tampere University of Applied Sciences, Kuntokatu 3, FI 33520 Tampere, Finland.

M. Mäntysalo is with the Department of Electronics and Communications Engineering, Tampere University of Technology, P.O. Box 692, FI 33101 Tampere, Finland

\section{INTRODUCTION}

TKJET-printed electronics (IPE) is one potential additive manufacturing solution in current and future low-cost electronics due to its material efficiency, ease of production and customization, low cost of processing equipment, and environmental aspects [1-3]. Some other considerable additive manufacturing technologies are material extrusion, powder bed fusion, binder jetting, vat photo-polymerization, directed energy deposition, and sheet lamination [4].

The rather low processing temperatures of advanced silver inks of around $150{ }^{\circ} \mathrm{C}$ enable the use of a wider scale of materials and structural solutions than, for example, with traditional printed circuit board technology with surface mount devices [5,6]. The minimum width of inkjet-printed lines is defined by the drop volume, which in commonly used modern technology can be around $1 \mathrm{pl}$. This results in a drop diameter of about $25 \mu \mathrm{m}$ on the substrate, depending on the ink, the surface, and the use of surface modifiers [7]. State-of-the-art technology (Electrohydrodynamic EHD printing) suggests that a scale of 0.1 femtoliter is available, which is capable of producing line widths on a micrometer scale [8]. In addition to the aforementioned factors, the electrical conductivities of silver inks enable their use in a wide variety of applications reaching up to the $\mathrm{GHz}$ range [9-11].

In this research, inkjet printing was used to fabricate an RFID tag's radiator traces on planar substrates, simultaneously with interconnections, with the so-called print-on-slope technique, for RFID chips. The operating band of the chips was in the ultra-high-frequency (UHF) range, at $865-868 \mathrm{MHz}$. While inkjet-printing of RFID antennas has been demonstrated earlier by various research groups, fabrication of the entire tag structure including the component interconnection is a new technique $[12,13]$. This was complemented by another test set with planar conducting traces for observing the effect of stressing in the cured ink.

To characterize reliability, the fabricated test structures were put into a thermal/humidity test chamber ("85/85" test) and their read ranges were measured intermittently during test breaks. Finally, SEM imaging was used to characterize the samples, including crack locations in the structures and qualitative analysis of the prints. The basic inkjet-printed lines were measured and visually inspected during test breaks to 
observe how the resistances and adhesion of silver ink on different substrates are affected by an elevated thermal/humidity environment.

Figure 1a shows a sketch of RFID chip attachment (combining silicon-on-flex and print-on-slope concepts [1416]) and the inkjet-printed silver lines of the presented structure. The manufactured structure whose design was adopted from an industrial partner is presented in Figure 1b, where also the bendability of the tag is demonstrated.

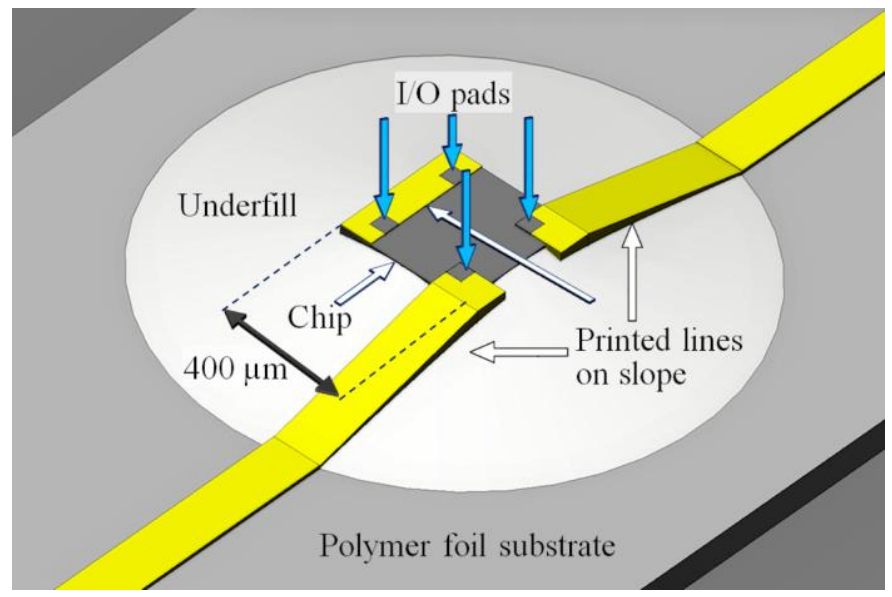

a)

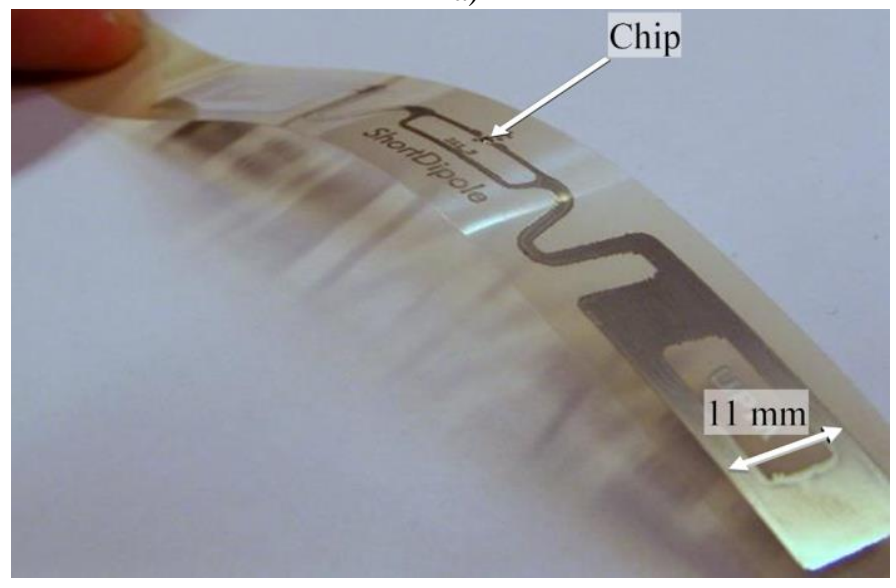

b)

Figure 1.a) Sketch of RFID chip attachment, also showing the print-on-slope concept. b) Manufactured RFID tag, consisting of RFID chip and inkjetprinted $\mathrm{Ag}$ on a polymer substrate.

\section{EXPERIMENTAL}

\section{A. Materials and sample manufacturing}

The RFID chips used in the tags were provided by UPMRaflatac. The size of the chip is $450 \mu \mathrm{m} \times 450 \mu \mathrm{m} \times 150 \mu \mathrm{m}$. Si has a coefficient of thermal expansion (CTE) of about 2.6 $\mathrm{ppm} /{ }^{\circ} \mathrm{C}$.

A commercially available silver nanoparticle ink from Harima (NPS-JL, Harima Chemicals, Japan) was used to inkjet-print the antenna and the RFID chip connections. NPS$\mathrm{JL}$ is an n-tetradecane-based nanoparticle dispersion which has 55 weight- $\%$ of silver particles with a 7 -nm diameter.
Three substrate materials were used. Polyimide (PI, 3M Kapton 500HN, USA) was included in the study as it is one of the most commonly used substrate materials in printed electronics. Polyethylene terephthalate (PET, 3M Melinex ST506, USA) and poly(p-phenylene oxide) (PPO)/poly(pphenylene ether) (PPE) (Premix, Finland) substrate were also tested to demonstrate fabrication capability on less expensive substrate materials, which on the other hand have lower glass transition temperatures $(\mathrm{Tg})$, and to review their performance in the test. The substrate materials are listed in Table I together with the thicknesses and glass transition temperatures given by the manufacturers. In addition to this data, according to the manufacturers, moisture absorption of the PI and PPO/PPE substrates are $4 \%$ and $0.2 \%$, respectively, whereas the manufacturer of PET substrate gives the coefficient of hygroscopic expansion as 6 parts-per-million ( $\mathrm{ppm}$ ) per percent of relative humidity (R.H.) between $40 \ldots 80 \%$ R.H.

TABLE I

SUBSTRATE MATERIALS USED IN THE RESEARCH

\begin{tabular}{lccl}
\hline \hline \multicolumn{1}{c}{ Substrate material } & $\begin{array}{c}\text { Nominal } \\
\text { thickness }\end{array}$ & $\begin{array}{c}\mathrm{Tg} \\
\left({ }^{\circ} \mathrm{C}\right)\end{array}$ & $\mathrm{CTE}\left(\mathrm{ppm} /{ }^{\circ} \mathrm{C}\right)$ \\
\hline $\begin{array}{l}\text { Polyimide (PI) } \\
\begin{array}{l}\text { Polyethylene } \\
\text { terephthalate (PET) }\end{array}\end{array}$ & $125 \mu \mathrm{m}$ & 360 & $17-20$, at $-14-100{ }^{\circ} \mathrm{C}$ \\
$\begin{array}{l}\text { Poly(p-phenylene } \\
\text { oxide)/poly(p-phenylene } \\
\text { ether) (PPO/PPE) }\end{array}$ & $125 \mu \mathrm{m}$ & 80 & 10, at $20-50{ }^{\circ} \mathrm{C}$ \\
\hline \hline
\end{tabular}

The RFID tags were fabricated in a so-called "component first" method in which the component was first attached to the substrate and the antenna and component connections were printed on top of the component [17]. The RFID chip was attached to the substrate using Epotek U300 (Epoxy Technologies, USA) adhesive material with a CTE of 55 $\mathrm{ppm} /{ }^{\circ} \mathrm{C}$ below the glass transition temperature of $127^{\circ} \mathrm{C}$. This epoxy underfill was placed onto the substrate with a manual dispenser. The adhesive was thereafter cured for 10 minutes at $150{ }^{\circ} \mathrm{C}$ according to the manufacturer's instructions.

The antenna structure and component interconnections were printed with an iTi XY 2.0 MDS prototype inkjet printer. A nominal resolution of 5050 drops per inch (dpi) was used, but a masking algorithm employed to improve drying of the inkand thus print quality-meant that the actual effective resolution was 789 dpi. The masking algorithm is explained in detail in ref [18]. Spreading of the ink was also controlled by using a solution of $1 \%$ EGC-1720 (3M, USA) surface energy modifier on the substrate prior to printing [19]. After printing, the silver nanoparticle ink was sintered for 60 minutes at 150 ${ }^{\circ} \mathrm{C}$ according to the manufacturer's instructions. Altogether 26 RFID tags were manufactured, verified to be operational, and put to the test. These tags consisted of ten with PI, nine with PET, and seven with PPO/PPE substrates. Figure $1 b$ shows an example structure with PPO/PPE substrate.

In addition, inkjet-printed lines were manufactured and their resistances measured on all the substrate types (24 lines per substrate type) to observe solely the response of inkjet-printed 
lines to stressing. Examples of Ag lines on PI, PET, and $\mathrm{PPO} / \mathrm{PPE}$ substrates are shown (after stress test) in Figure 2a$c$, respectively. Some extraneous spreading of ink on each of the substrates can also be observed.

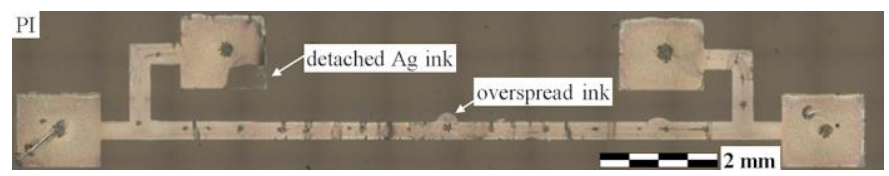

a)

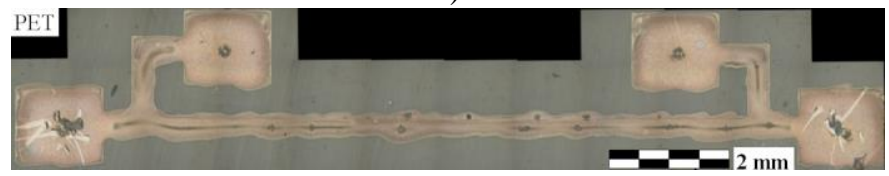

b)

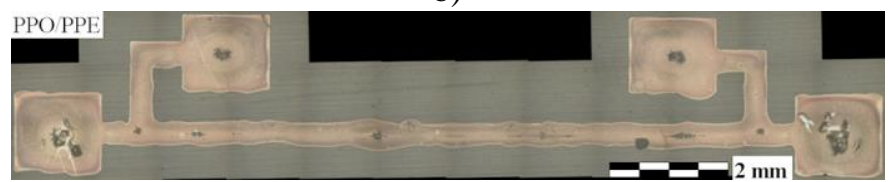

c)

Figure 2. Laser microscopy images of the design used in simple resistance monitoring of cured ink during the test breaks. Images taken after stress test from prints on a) PI, b) PET, c) PPO/PPE substrates.

\section{B. Stress tests}

Moisture absorption of polymer materials can be a challenge to reliable operation of electronics. Therefore, to characterize the reliability of the RFID tags, an "85/85" temperature/humidity $(\mathrm{T} / \mathrm{H})$ life test was used [20]. The standard defines the temperature as $85 \pm 2{ }^{\circ} \mathrm{C}$ and relative humidity as $85 \pm 5 \%$. The test time is defined as at least 1000 hours. The $85 / 85$ accelerated stress test was chosen to study whether or not-besides elevated temperature - the moisture absorption of polymer affects the reliable operation of printed RFID devices.

The total stress time for the printed RFID tags was 1724 hours and the tags were taken out for inspection nine times. The stress time between inspections varied from 139 to 353 hours, while the median stress interval was about 160 hours. The bulk conductors were measured during the same intervals; however, their stressing was continued up to 2007 hours.

\section{Characterization methods}

Before the tests and during the stress intervals the RFID tags were inspected visually and their functionality verified. Functional verification, presented in detail in ref. [21], was done using a Voyantic Tagformance lite RFID test chamber (Voyantic, Finland) to make sure each of the RFID tags responded to the RFID transponder. Changes in read ranges were characterized during the stress test intervals by contrasting those measurements to the initially measured read ranges. No specific read range threshold for determining a tag as failed was used, but it was deemed failed when it did not respond to the transponder at all.

Resistances of basic conductive lines were measured during the test breaks to observe the response of the cured ink to stressing.

Imaging of the samples was done with an optical microscope and a (field emission) scanning electron microscope (FE-SEM). In addition, laser microscopy was used to support these imaging methods. Optical microscope images were taken from the top of the samples to observe the inkjetprinted interconnections and traces. SEM images were taken from a slight angle near the top of the samples to observe especially the interconnection area of the conductor traces near the RFID chips. Selected samples were immersed in an epoxy resin and dried, followed by careful grinding and polishing to reveal the cross-sections of the RFID structures.

\section{RESULTS}

\section{A. Read range measurements of RFID structures}

Before stress testing, the measured read ranges averaged 5.8 $\mathrm{m}$, with a range of 4.4-7.0 meters. The data related to the read range measurements of the structures on each substrate are presented in Table II.

TABLE II

TEST SET DATA

\begin{tabular}{lccc}
\hline \hline & \multicolumn{3}{c}{ Substrate material } \\
Property & PI & PET & PPO/PPE \\
\hline Number of samples & 10 & 9 & 7 \\
Broken after test (1724 hrs.) & 7 & 6 & 1 \\
Average read range (m) & 6.21 & 5.45 & 5.70 \\
$\quad$-Standard deviation (m) & 0.66 & 0.42 & 0.81 \\
Average change in read range (m) & 0.11 & 0.32 & 0.35 \\
& & & \\
$\begin{array}{l}\text { Amount of samples with increased } \\
\text { read range in the end compared to start }\end{array}$ & 1 & 7 & 4 \\
\hline \hline
\end{tabular}

Examples of read range measurement results of RFID tags obtained during intervals of the test is presented in Figure 3a-c. No remarkable degradation in the read ranges has occurred in these case after 1724 hours of testing. In Figure 3b and $\mathrm{c}$, the maximum read range has actually increased at the end compared to the start of the tests, being as much as 1.291 $\mathrm{m}$ for the PPO case, Figure 3c. This behavior was observed with 7 out of 9 and 4 out of 7 of the samples printed onto PET and PPO/PPE substrates, respectively. With PI substrates the last read range measurements, taken either before breakdown of the sample or at the end of the test, were lower than the initial values in all except one PI sample (also, one PI sample was broken during the first stress test interval, between 0-164 hours). For the failed tags the change from functioning to failed was sudden, i.e. it occurred within one testing interval, and no indications of failures were observed beforehand. 


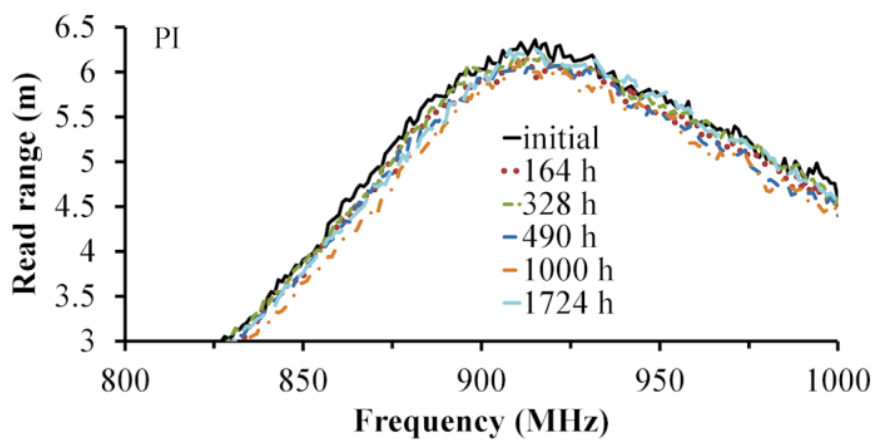

a)

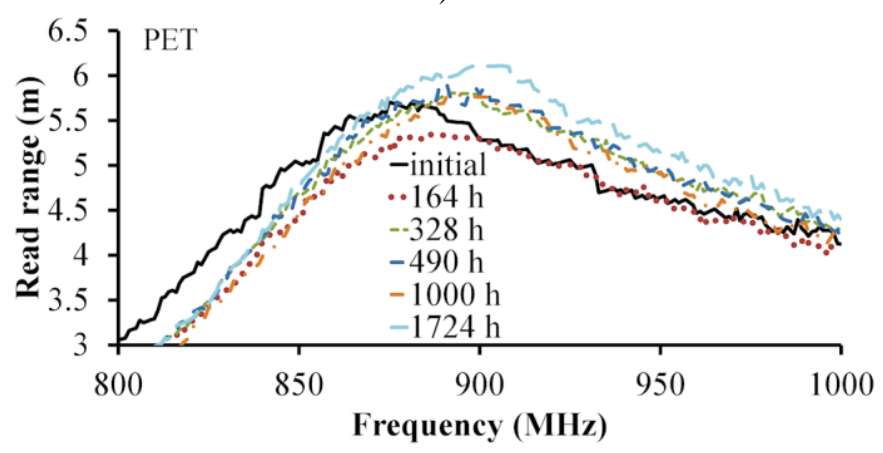

b)

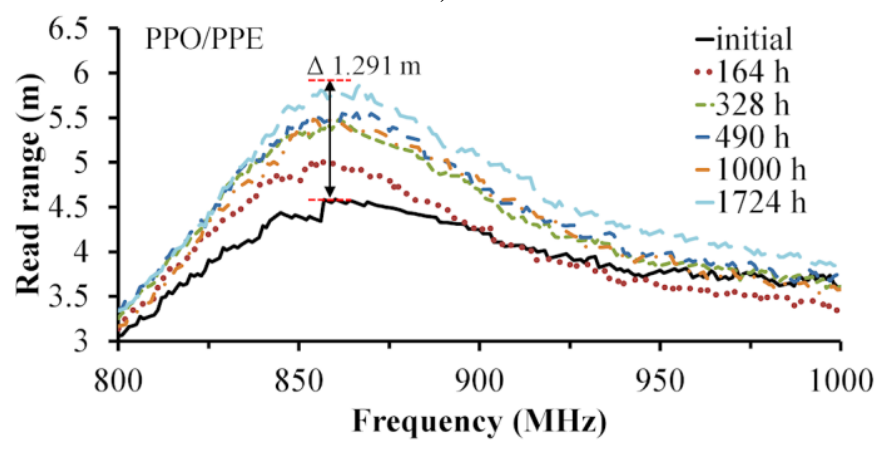

c)

Figure 3. Read range measurement results of RFID tags at different phases of the test, showing the peak read range in the operating band. a) PI, b) PET, c) PPO/PPE.

On the basis of the read range measurements, Figure 4 shows the percentual amount of failed RFID tags as a function of time periods of stress testing where the tag has failed. The figure suggests that the tags using PPO/PPE as a substrate material had better reliability with no failures after 1000 hours of testing, and one failed tag out of seven after the test, thus indicating that $14 \%$ of the population failed, while tags with PI and PET as a substrate were somewhat less reliable.

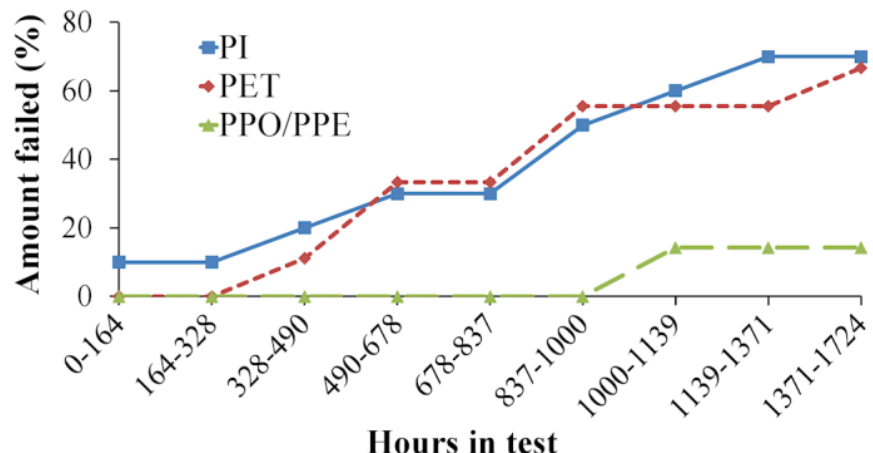

Figure 4. Amount of failed RFID tags as a function of test time.

\section{B. Resistance measurements of inkjet-printed conductor lines}

Resistance measurements of the printed lines were done on each of the three substrate types. Two individual samples of each substrate type were used, with 12 lines printed on both, i.e. 24 lines per substrate type were measured. The averaged resistances and standard deviations of the lines on each substrate are presented in Figure 5. For lines printed on PET and PPO/PPE substrates, clearly decreasing trends in the averaged resistance values can be seen, whereas for the PI substrate, this trend does not exist. The average standard deviations of the resistances in the whole testing period were 1.1 and 0.42 for substrates PI_1 and PI_2, respectively; 0.17 and 0.14 for PET_1 and PET_2, respectively; and 0.14 and 0.12 for PPO/PPE_1 and PPO/PPE_2, respectively. The adhesion of the printed conductors to the PI substrates was found to be poor.

During the testing a total of six printed resistance measurement lines failed during four intervals, all on PI substrates, see Figure 5a. In the figure, points i)-iv) show failures that occurred between i) 490-678 hours, ii) 678-837 hours, iii) 1000-1139 hours, and iv) 1724-2007 hours, respectively.

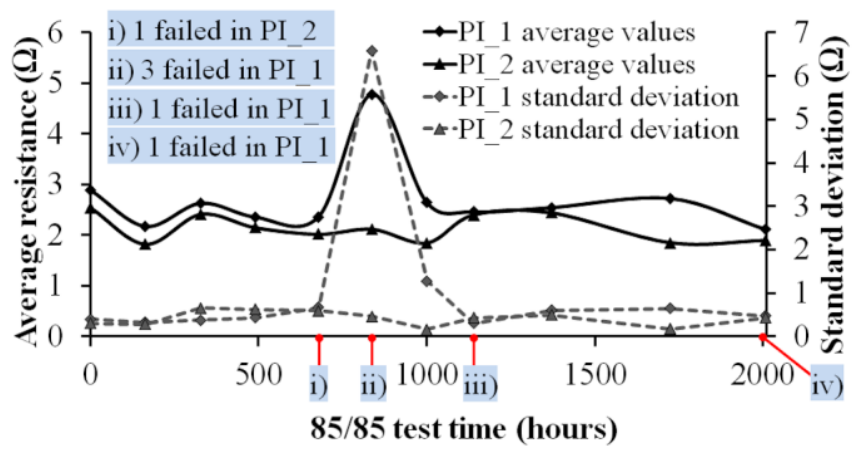

a)

Copyright @ 2016 IEEE. Personal use of this material is permitted. However, permission to use this material for any other purposes must be obtained by sending a request to pubs-permissions@iee.org 


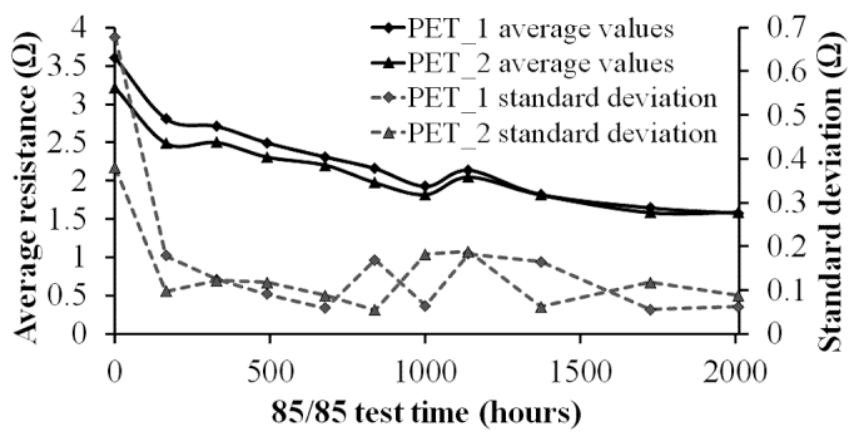

b)

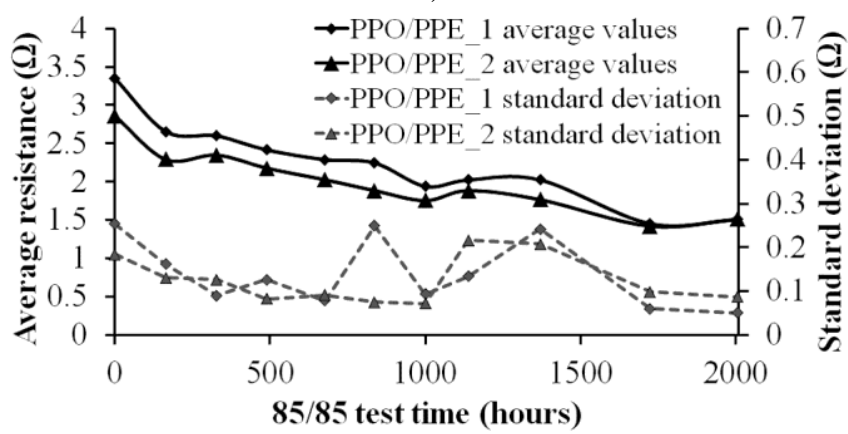

c)

Figure 5. Average resistance values and standard deviations of inkjet-printed conductor lines on a) PI, b) PET, c) PPO/PPE substrates.

\section{Optical and SEM imaging}

Figure $6 \mathrm{a}$ is a top view of the chip attachment area on a substrate showing underfill, RFID chip, print-on-slope, and the interconnection parts of the silver lines to the chip. Figure $6 \mathrm{~b}$ and Figure $6 \mathrm{c}$ show problems encountered during manufacturing, i.e. non-uniform spreading of the ink and misalignment of the chip on top of the underfill. Despite the somewhat poor quality, these particular RFID tags were functional. Besides these initial process problems, in some cases the ink spread along geometrically favorable features of the chip.

Figure 6d shows the initial and after test conditions of a test case with PET substrate, respectively. The stress-induced cracks are seen most clearly in the corner area pointed out with an arrow.

Complementary laser microscopy imaging and measurements showed the ink thickness of conductors being on the order of 3 $\mu \mathrm{m}$.

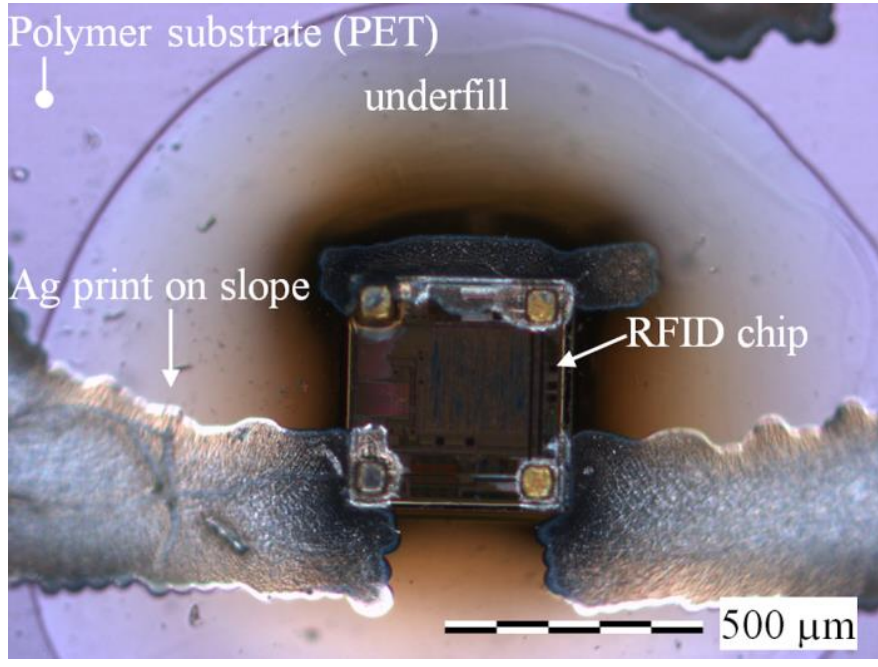

a)

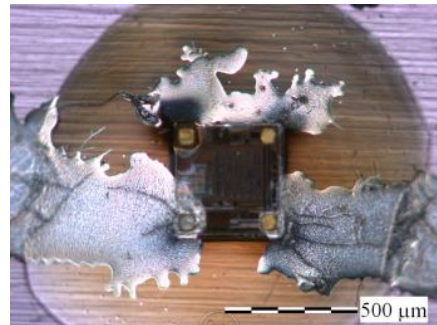

b)

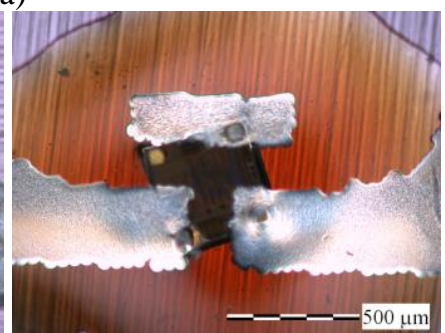

c)

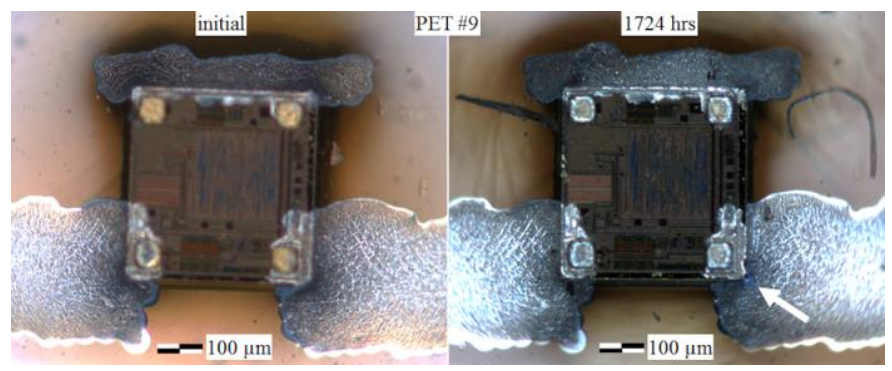

d)

Figure 6. Images taken from top of RFID tags. a) RFID chip attached with underfill onto a PET substrate, together with inkjet-printed Ag lines shown. b) Non-uniform distribution of $\mathrm{Ag}$ ink on underfill deposited on top of a PPO/PPE substrate. c) Misaligned component in the underfill deposited on top of a PPO/PPE substrate. d) PET sample \#9 before stress test (left) and after 1724 hours of $85 / 85$ test (right).

Figure 7 is a cross-section image of a stress-tested RFID tag with a PPO/PPE substrate, showing a side view of the printon-slope structure and ink spreading. The ink thickness is slightly smaller on top of the chip than on the slope part, i.e. the underfill.

SEM micrographs from the top of the RFID tags show the quality of printing, some observed anomalies, and stressinduced cracks (Figure 8a-c). Observed thickness of the Ag print was on the order of $3 \mu \mathrm{m}$ in SEM images as well.

Figure $8 \mathrm{a}$ shows a stressed sample with some, yet noncritical, process issues. These include grooves in the thickest part of the Ag layer on top of the chip, and one interconnection trace having an impaired connection to the pad due to slightly misaligned print combined with extraneous flow of underfill material on top of the chip corner area. In spite of these

Copyright @ 2016 IEEE. Personal use of this material is permitted. However, permission to use this material for any other purposes must be obtained by sending a request to pubs-permissions@ieee.org 
defects, the tag was operational before stress testing: initially the read range was $6.54 \mathrm{~m}$, which was better than the average of the test set $(6.21 \mathrm{~m})$. The imaging of the structure was performed after testing. Looking at the lower left side in more detail, inset of Figure 8a, an area of cracked silver print can be seen, with a crack opening being most significant in the corner of the chip. This (PI) sample was the first broken during the testing, between 0-164 hours, confer to Figure 4.

Figure $8 \mathrm{~b}$ shows a common failure observed in the structures, i.e. cracking of the chip-underfill interface, which has cracked the silver ink, as well. Besides this, the figure shows how silver ink has run along the vertical details on top of the chip (upper part of the image) during processing. Cracks similar to those shown in Figure 8a-b were observed with all three substrate materials after stress test. Figure $8 \mathrm{c}$ shows the cracked corner area of a sample with PET substrate after 1724 hours of $\mathrm{T} / \mathrm{H}$ test, pointed out with an arrow in the optical microscope image, Figure 6d.

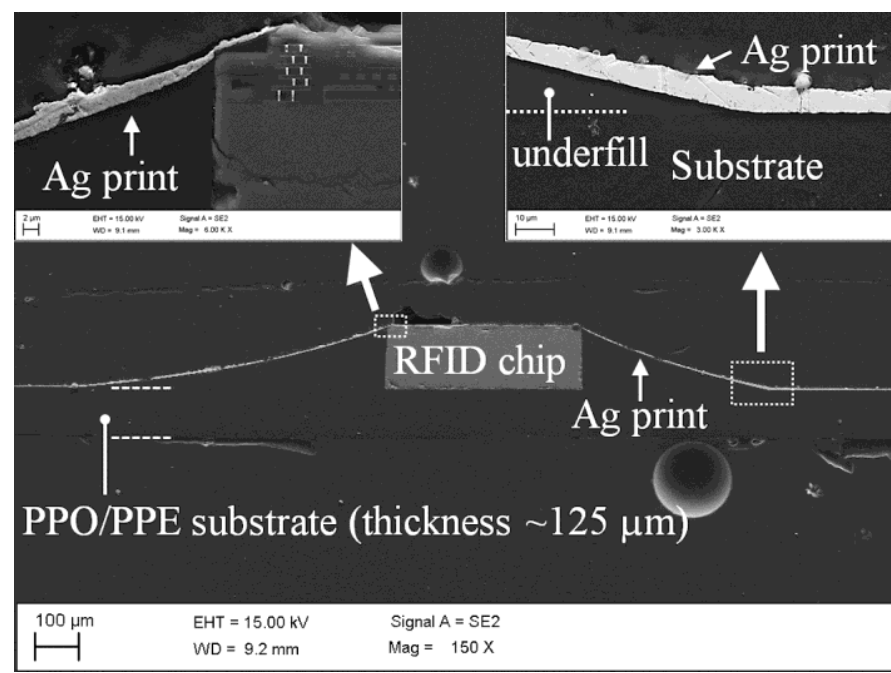

Figure 7. Cross-section view of an RFID chip attachment on a PPO/PPE substrate, showing the result of the print-on-slope technique; images were taken from a stressed sample after 1139 hours in the 85/85 test.

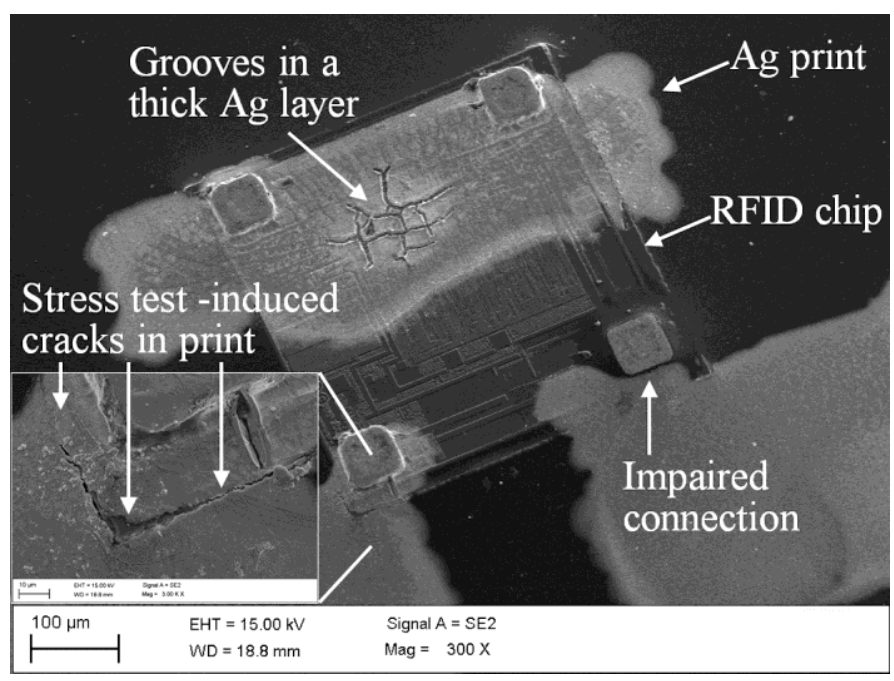

a)

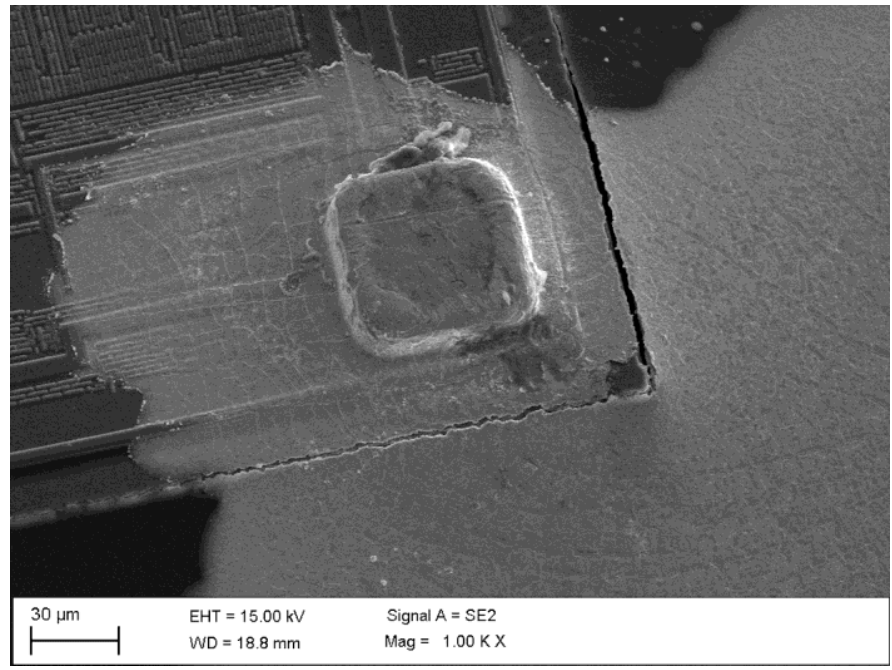

b)

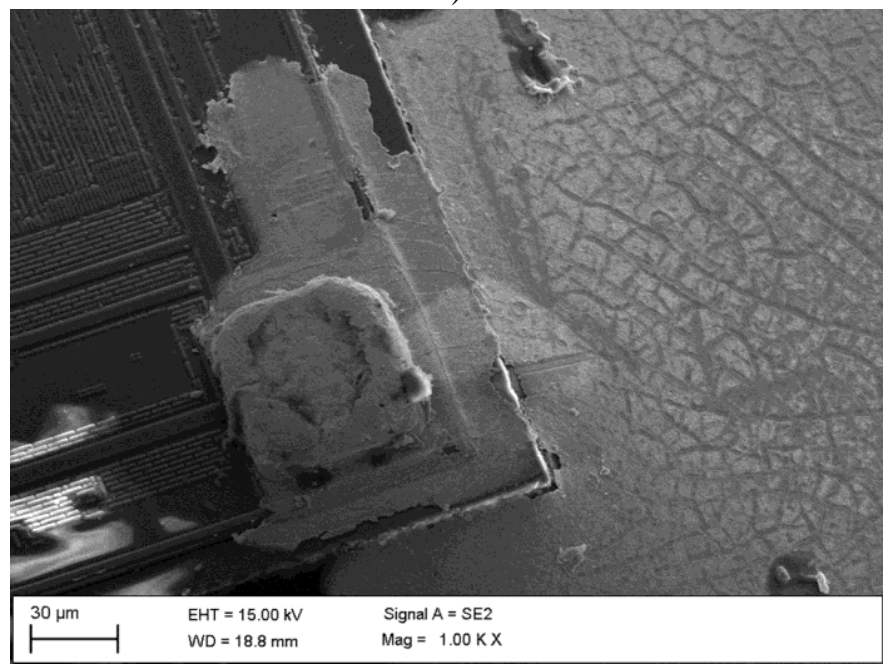

c)

Figure 8. a) Ag print on the chip and in its vicinity (PI substrate) with a inset image of the lower left corner, showing stress test induced cracks in print. b) 1000x magnification of a chip's corner, showing cracking of the underfillchip interface of a chip attached on PI, which has resulted in cracking of the conductor. c) Test induced cracks in print, PET substrate.

\section{DISCUSSION}

\section{A. Process}

\section{1) Print-on-slope technique}

The print head was kept at a fixed location during the processing and the "focal point", in a sense, was adjusted according to the chip structure. This resulted in the actual print distance being smaller on top of the chip compared to the substrate. However, no significant difference was observed in the resulting print.

The main challenge in the printing phase was optimization of the surface energy of the substrate, especially the slope. Wetting modification is necessary as the silicon chip, the slope material, and the polymer substrates have different surface characteristics.

As Figure 6b shows, a potential risk that should be noted in manufacturing printed interconnections is the ink spreading along the surface of the chip, adding to the possibility of short-

Copyright $\odot 2016$ IEEE. Personal use of this material is permitted. However, permission to use this material for any other purposes must be obtained by sending a request to pubs-permissions@ieee.org 
circuits between connecting pads. However, robustness of the design ensured the functionality of the RFIDs despite process fluctuations.

In some cross-sections ink thickness was slightly smaller on the chip compared to the slope and substrate parts, see Figure 7. The causes of this can be i) in the edge areas of the print, such as on the chip, the ink is thinner than in the middle areas, and ii) the ink had flowed downhill from the top part of the underfill during the process.

\section{2) Ink curing temperatures}

Post-curing of the silver ink was observed in resistance measurements of the lines printed on the PET and PPO/PPE substrates. This can be seen in Figure 5b-c, where the average resistance values were reduced to approximately half of the initial average value during the execution of the whole test. This is in line with previous results [22]. Contrary to this, no such phenomenon was observed with the PI substrate. Although an increased state of curing of ink printed on PI substrates cannot be ruled out, a more likely factor is the adhesion problem, which caused a large deviation in the measured resistance values, thus masking the reduction in resistance.

\section{B. Measurements and reliability test results}

\section{1) Read range}

It was found with each tag that stress testing did not reduce the measured read range significantly until a total failure occurred. In most cases with PET and PPO/PPE substrates the read range was actually higher at the final measurement before breakdown or at the end of the test; an example is shown in Figure $3 \mathrm{c}$. This occurred with only one sample with PI. This increase hints towards post-curing of the ink during the stress test. This phenomenon has been observed earlier [22].

Decreasing ohmic resistance can be straightforwardly linked to improving read distance. In free space, the read range can be presented by Friis' equation, see [23], for example. The equation in its basic form is

$$
\frac{P_{r}}{P_{t}}=G_{t} G_{r} \cdot\left(\frac{\lambda}{4 \pi r}\right)^{2}
$$

where

$P_{r} \quad=$ received power

$P_{t} \quad=$ transmitted power

$G_{t} \quad=$ gain of the transmitting antenna: $G_{t}=\eta_{t} \cdot D_{t}, \eta_{t}=$

efficiency; $D_{t}=$ directivity of the transmitting antenna

$G_{r} \quad=$ gain of the receiving antenna

$\lambda=$ wavelength of the RFID's operation frequency

$r \quad=$ distance of the transmitting and receiving antennas (maximum read range of the RFID tag)

Rearranging the equation to find the read range, and including power reflection coefficient $|s|^{2}$ presented by Kurokawa [24] results in

$$
\begin{aligned}
& r=\frac{\lambda}{4 \pi} \sqrt{\frac{P_{t} G_{t} G_{r}\left(1-|s|^{2}\right)}{P_{r}}} \\
& \text { where } \\
& |s|^{2}=\left|\frac{Z_{L}-Z_{S}^{*}}{Z_{L}+Z_{S}}\right|^{2}, \quad 0 \leq|s|^{2} \leq 1
\end{aligned}
$$

the rest parameters being

$|s| \quad=$ power wave reflection coefficient

$Z_{L} \quad=$ load impedance (e.g. tag's RFID chip)

$Z_{S} \quad=$ source impedance (e.g. tag's RFID antenna), asterisk

implies a conjugate

The ratio of output and input powers, $P_{r} / P_{t}$, represents the efficiency $\eta$ of the whole transmission path. Setting aside the reflections from mismatches, this number is affected by gains of antennas (which accounts for efficiency of both antennas), wavelength of the operating frequency, and distance between antennas as pointed out in Figure 9. The figure shows reference antenna patterns as spheres, and main lobes of the actual antennas with directivities $D_{t}$ and $D_{r}$ as cones pointing towards each other. Often a high directivity is desired so that the antenna can transmit/receive signal at a given direction from far away. Gains $G_{t, r}$ account for directivities and also efficiencies $\eta_{t, r}$, affected by losses such as resistive loss $R_{\text {loss. }} \theta$ and $\Phi$ are the elevation and azimuthal angles ranging from $0 \ldots \pi$ radians $\left(0 \ldots 180^{\circ}\right)$ and $0 \ldots 2 \pi$ radians $\left(0 \ldots 360^{\circ}\right)$, respectively. They are used in representing the radiation patterns of antennas, $F(\Theta, \Phi)$ function values, in spherical coordinate system.

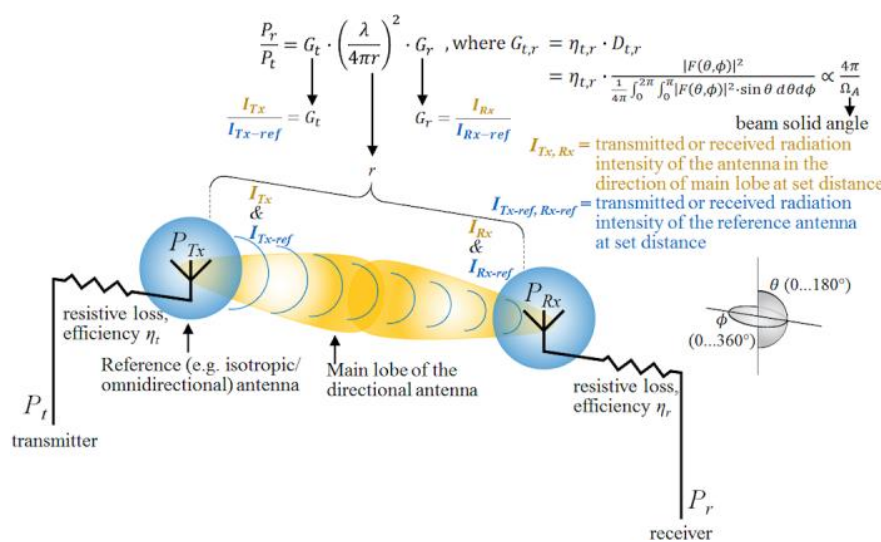

Figure 9. Transmission path from transmitter to receiver.

Looking at a single (transmitting) antenna element, similarly the proportion of antenna's radiated versus applied input power, $P_{T_{X}} / P_{t}$, shows its efficiency [25]. Reciprocally, for the receiving antenna the proportion of its available output and input powers $\left(P_{r} / P_{R x}\right)$ is equivalent to its efficiency. To simplify following analysis it is assumed that good impedance matching exists for operation (i.e. resonance) frequency, and therefore practically no power is reflected, $|s|^{2} \rightarrow 0$, and gain equals realized gain. Thus, the power received at the element is either transferred onwards or dissipated by ohmic losses $R_{\text {loss }}$ along the trace. Considering the power depleted through ohmic loss and that $P=I^{2} R$, the efficiency $\eta_{\text {tag }}$ expressed by terms of 
power and finally by proportionality of resistances (e.g. [26]) is

$$
\eta_{\text {tag }}=\frac{P_{r}}{P_{R x}}=\frac{P_{r}}{P_{r}+P_{\text {loss }}} \propto \frac{R_{r}}{R_{r}+R_{\text {loss }}}
$$

where

$P_{r} \quad=$ power delivered to the tag's chip, see equation (1)

$P_{R x} \quad=$ input power to the tag's radiator

$P_{\text {loss }} \quad=$ power loss due to ohmic resistance

$R_{r} \quad=$ antenna's radiation resistance

$R_{\text {loss }}=$ antenna's loss resistance

Figure 10 presents the RFID tag in receiving mode. For calculations of e.g. the required transmission power, $P_{r}$ could be chosen to be the threshold power needed to power up the RFID chip.

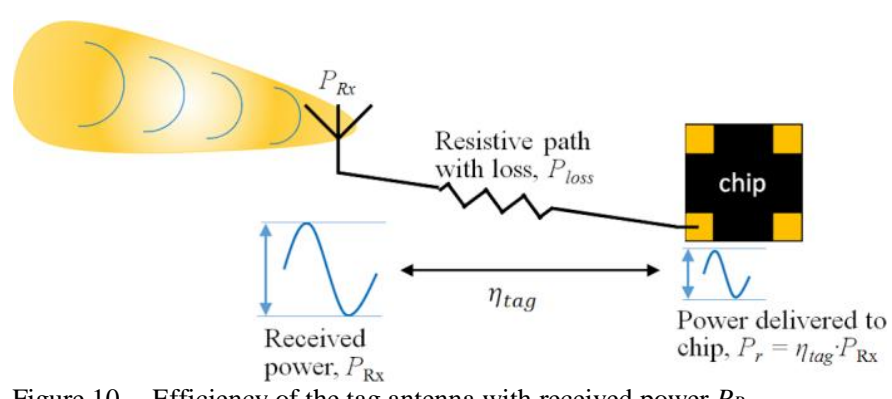

Figure 10. Efficiency of the tag antenna with received power $P_{R x}$.

Looking at equation (4) and Figure 10, large $R_{\text {loss }}$ compared to $R_{r}$ results in low antenna efficiency $\eta_{\text {tag }}$, i.e. large portion of power is attenuated/transformed into heat along the conductor. Therefore, maximizing antenna efficiency requires maximizing the proportion of radiation resistance $R_{r}$ to loss resistance $R_{\text {loss }}$. With current case, decreasing $R_{\text {loss }}$ due to post-curing of the inkjet-printed lines results in increasing efficiency $\eta_{t a g}$, and therefore an increased read range $r$.

As stated before, measurements were made during the testing intervals. However, slightly larger variation could occur before failure if the measurements were made during stressing. This has been noted previously with $\mathrm{DC}$ and RF measurements of interconnections, and is discussed, for example, in refs. [27, 28]. On the basis of a short search, the read ranges of inkjet-printed tags are close to or equal to those made with traditional technology with etched aluminum or copper traces. When the fractures on traces connecting the chip to the radiator have propagated through the whole interconnection, as most clearly shown in Figure 8b, an electrical discontinuity occurs and the tag fails to respond to the reader.

\section{2) Resistance measurements of bulk conductors}

As mentioned in the results, the average resistances of the lines printed on PET and PPO/PPE substrates were reduced during the execution of the test. This was likely due to postcuring of the ink. Similar results have been found with paper substrates [29]. In addition to the measurements being performed at room temperature, the resistances were not continuously monitored during the stressing, which might have masked some of the failures. No indications of failures were observed beforehand in the resistance measurements. Poor adhesion of the ink onto the PI substrate caused randomness in the measured resistances. This effect can be seen by comparing the standard deviations of each substrate in Figure 5. This finally resulted in failures of some of the conductors. Disregarding these also easily visually observable failures it can be noted that the ink-substrate interface was not affecting the reliability lifetime of the actual RFID test case, and the critical place concerning reliability is elsewhere (chip-underfill interface).

\section{3) Differences between substrates}

Although the amount of samples in the tests was not large, the results can be considered indicative. Of the samples, the ones printed on PPO/PPE substrates seem to be most reliable under combined thermal and humidity stress. With PI the adhesion of the ink was found to be a challenge. Peeling of the printed lines was observed in many cases. For example, by roughening the rather smooth substrate surface the ink would have adhered to it better. However, this result together with previous supporting results, and also noting the large moisture absorption of PI, [22, 30] suggests that the use of this inksubstrate material combination is rather risky.

\section{4) Location of cracks}

On the basis of SEM imaging, the most vulnerable place on the RFID tags is the chip corner areas, at the interface between the chip and underfill, as seen in Figure 8a-c. The reason for this is that the materials (Si chip versus polymer substrates) are dissimilar in terms of both thermal expansion and moisture absorption. Si has a CTE of about $2.6 \mathrm{ppm} /{ }^{\circ} \mathrm{C}$ as noted earlier, and in addition it does not absorb moisture, whereas the CTEs of polymer substrates and underfill are many times highersee Table I-and are prone to moisture absorption.

This research showed that $85 / 85$ tests induced cracks in the chip-underfill interface, finally breaking the interconnections to the silicon chip's pads. This cracking was not observed with non-stressed sample. No cracks were anticipated nor observed in the functional areas of the chips. The polymer material absorbed humidity and swelled, this together with the elevated temperature caused creep that finally resulted in electrical opens in the printed traces. In addition to this, delamination of the printed conductors from their substrates was observed with PI, suggesting incompatibility between the ink and the smooth and inert surface of PI.

It is a known fact that increasing the distance to neutral point (DNP) increases stresses in interconnections. Thus, using a larger chip increases stresses at the vertical chip-underfill interfaces, i.e. the edges and corners of the chip, resulting in earlier failures. Therefore, with the current configuration the chip size of $450 \times 450 \mu \mathrm{m}$ is near, if not, the maximum, size that can be used with a chip thickness of $150 \mu \mathrm{m}$. Increasing the chip thickness and thus the interconnection height will not likely increase reliability due to increased rigidity and the different properties (CTE and moisture absorption in this case) of the chip compared to the substrate and underfill. The problematic vertical interface remains the same, and may not 
therefore be contrasted to the more common case of interconnection height in solder interconnections, for example.

\section{Stress testing}

In this study, 85/85 $\mathrm{T} / \mathrm{H}$ stress testing was selected in order to observe the behavior of the samples, especially the printed interconnections at elevated temperature and humidity. Moisture absorption of polymer materials can cause problems in a field environment, as can elevated temperatures, which both in this case caused stresses in the interfaces of the different materials. Also, potential adhesion problems between the inkjet-printed, low-temperature curable silver conductors and substrates were of interest, as these issues have been noted earlier in stress tests with PI substrates and inkjet-printed silver conductors [22, 31, 32].

The test duration recommended in the JEDEC A-101C standard is 1000 hours. In the current research, however, the stressing was continued for up to 2000 hours in order to record more failure times of the samples, and thus to better determine their reliability.

\section{CONCLUSIONS}

This work presented a capability assessment of inkjet printing for reliable RFID applications. 85/85 tests were used to induce stresses in the manufactured flexible RFID tags. Also simpler structures made of similar conductor and substrate materials were used in the tests for monitoring resistance changes of the actual inkjet-printed lines. Characterizations (both electrical and imaging) were performed on the structures. According to the results, the following conclusions can be made:

1) The material and structural compatibilities of inkjetprinted and more traditional electronics manufacturing technologies are crucial factors affecting reliability. These issues can be condensed into three factors: adhesion, thermomechanical properties, and dimensions.

2) Challenges during IPE processing that must be considered include spreading of ink, material thickness control, component attachment, and curing profile optimization.

3) The print-on-slope technique is a potential additive manufacturing solution for connecting pads of small chips successfully onto thin substrates in one phase process.

4) Post-curing of silver ink can occur in spite of following a conservative ink curing profile. This was observed in electrical measurements as a decreasing resistance value, which related to increasing RFID read range.

5) Read range measurements made during testing intervals showed no degradation before total failure of RFID tags. In best case, the read range actually improved as much as $1.3 \mathrm{~m}$ due to post-curing of the ink during stressing.

6) Of the tested material and structural combinations, the one with PPO/PPE substrate passed the 1000-hour no-fault test requirement in the $85 / 85$ test.

\section{ACKNOWLEDGMENT}

The authors would like to thank M.Sc. Tuomo Siponkoski from the University of Oulu for laser microscopy imaging and measurements.

\section{REFERENCES}

[1] A. C. Siegel, S. T. Phillips, M. D. Dickey, N. Lu, Z. Suo and G. M. Whitesides, "Printable Electronics: Foldable Printed Circuit Boards on Paper Substrates," Adv. Funct. Mater., vol. 20, pp. 28-35, 2010.

[2] B. S. Cook and A. Shamim, "Inkjet Printing of Novel Wideband and High Gain Antennas on Low-Cost Paper Substrate," IEEE T. Antenn. Propag., vol. 60, pp. 4148-4156, 2012.

[3] S. Wünscher, R. Abbel, J. Perelaer and U. S. Schubert, "Progress of alternative sintering approaches of inkjet-printed metal inks and their application for manufacturing of flexible electronic devices," J. Mater. Chem., vol. 2, pp. 10232-10261, 2014.

[4] B. P. Conner, G. P. Manogharan, A. N. Martof, L. M. Rodomsky, C. M. Rodomsky, D. C. Jordan and J. W. Limperos, "Making sense of 3-D printing: Creating a map of additive manufacturing products and services," Addit. Manuf., pp. 64-76, 2014.

[5] A. Kamyshny, J. Steinke and S. Magdassi, "Metal-based Inkjet Inks for Printed Electronics," Open Appl. Phys. J., vol. 4, pp. 19-36, 2011.

[6] S. K. Volkman, S. Yin, T. Bakhishev, K. Puntambekar, V. Subramanian and M. F. Toney, "Mechanistic Studies on Sintering of Silver Nanoparticles," Chem. Mater., vol. 23, pp. 4634-4640, 2011.

[7] V. Pekkanen, M. Mäntysalo, K. Kaija, P. Mansikkamäki, E. Kunnari, K. Laine, J. Niittynen, S. Koskinen, E. Halonen and U. Caglar, "Utilizing inkjet printing to fabricate electrical interconnections in a system-in-package," Microelectron. Eng., vol. 87, pp. 2382-2390, 11, 2010.

[8] M. Mashayekhi, L. Winchester, L. Evans, T. Pease, M. M. Laurila, M. Mäntysalo, S. Ogier, L. Terés and J. Carrabina, "Evaluation of Aerosol, Superfine Inkjet, and Photolithography Printing Techniques for Metallization of Application Specific Printed Electronic Circuits," IEEE Transactions on Electron Devices, vol. 63, pp. 1246-1253, 2016.

[9] V. Pynttäri, E. Halonen, H. Sillanpää, M. Mäntysalo and R. Mäkinen, "RF Design for Inkjet Technology: Antenna Geometries and Layer Thickness Optimization," IEEE Antenn. Wirel. Propag. Lett., vol. 11, pp. 188-191, 2012.

[10] S. Myllymäki, J. Putaala, J. Hannu, H. Jantunen, M. Mäntysalo and E. Kunnari, "Failure Mode Characterization In Inkjet-Printed CPW Lines Utilizing A High-Frequency Network Analyzer And Post-Processed TDR Analysis," Prog. Electromagn. Res., vol. 43, pp. 1-14, 2013.

[11] A. Chiolerio, V. Camarchia, R. Quaglia, M. Pirola, P. Pandolfi and C. F. Pirri, "Hybrid Ag-based inks for nanocomposite inkjet printed lines: RF properties," J. Alloy. Compd., vol. 615, Supplement 1, pp. S501-S504, 12/5, 2014.

[12] B. S. Cook, J. R. Cooper and M. M. Tentzeris, "An Inkjet-Printed Microfluidic RFID-Enabled Platform for Wireless Lab-on-Chip Applications," IEEE T. Microw. Theory, vol. 61, pp. 4714-4723, 2013.

[13] G. A. Casula, G. Montisci and G. Mazzarella, "A Wideband PET InkjetPrinted Antenna for UHF RFID," IEEE Antenn. Wirel. Propag. Lett., vol. 12, pp. 1400-1403, 2013.

[14] J. Haberland, B. Pahl, C. Kallmayer, R. Aschenbrenner and H. Reichl, "Super thin flip chip assemblies on flex substrates - adhesive bonding and soldering technology - reliability investigations and applications," in Proc. IMAPS International Symposium on Microelectronics, San Diego, CA, USA, 2006, pp. 8.

Copyright @ 2016 IEEE. Personal use of this material is permitted. However, permission to use this material for any other purposes must be obtained by sending a request to pubs-permissions@ieee.org 
[15] C. Banda, R. W. Johnson, T. Zhang, Z. Hou and H. K. Charles, "Flip Chip Assembly of Thinned Silicon Die on Flex Substrates," IEEE T. Electron. Packag. Manuf., vol. 31, pp. 1-8, 2008.

[16] G. Yang, L. Xie, M. Mäntysalo, X. Zhou, Z. Pang, L. D. Xu, S. KaoWalter, Q. Chen and L. R. Zheng, "A Health-IoT Platform Based on the Integration of Intelligent Packaging, Unobtrusive Bio-Sensor, and Intelligent Medicine Box," IEEE T. Ind. Inform., vol. 10, pp. 2180-2191, 2014.

[17] M. Mäntysalo, L. Xie, F. Jonsson, Y. Feng, A. Lopez Cabezas and Z. LiRong, "System integration of smart packages using printed electronics," in Proc. 62nd IEEE Electronic Components and Technology Conference (ECTC), 2012, pp. 997-1002.

[18] S. Koskinen, L. Pykäri and M. Mäntysalo, "Electrical Performance Characterization of an Inkjet-Printed Flexible Circuit in a Mobile Application," IEEE T. Compon. Packag. Manuf., vol. 3, pp. 1604-1610, 2013.

[19] M. Mäntysalo and P. Mansikkamäki, "Inkjet deposited interconnections for electronic packaging," in Proc. IST Digital Fabrication, Alaska, USA, 2007, pp. 813-817.

[20] JEDEC, "'Steady State Temperature Humidity Bias Life Test, JEDEC Standard JESD22-A101C"," 2009.

[21] J. Virtanen, T. Björninen, L. Ukkonen, K. Kaija, T. Joutsenoja, L. Sydänheimo and A. Elsherbeni, "The effect of conductor thickness in passive inkjet printed RFID tags," in IEEE Antennas Prop, Toronto, Canada, 2010, pp. 1-4.

[22] J. Putaala, J. Hannu, E. Kunnari, M. Mäntysalo, O. Nousiainen and H. Jantunen, "Reliability of SMD interconnections on flexible low-temperature substrates with inkjet-printed conductors," Microelectronics Reliability, vol. 54, pp. 272-280, 1, 2014.

[23] Sung-Jung Wu, Chi-Hung Chao and Jenn-Hwan Tarng, "A low-profile dipole type passive UHF band RFID tag antenna," in Antennas and Propagation (EuCAP), 2010 Proceedings of the Fourth European Conference On, 2010, pp. 1-4.

[24] K. Kurokawa, "Power Waves and the Scattering Matrix," IEEE Transactions on Microwave Theory and Techniques, vol. 13, pp. 194-202, 1965.

[25] H. G. Schantz, "Measurement of UWB antenna efficiency," in Vehicular Technology Conference, 2001. VTC 2001 Spring. IEEE VTS 53rd, 2001, pp. 1189-1191 vol.2.

[26] C. S. Lee, A. Duffy and C. Lee, "Antenna Efficiency Measurements in a Reverberation Chamber Without the Need for a Reference Antenna," IEEE Antennas and Wireless Propagation Letters, vol. 7, pp. 448-450, 2008.

[27] Institute of Printed Circuits (IPC), "IPC-9701 Standard: Performance Test Methods and Qualification Requirements for Surface Mount Solder Attachments," IPC Standard IPC-9701, 2002.

[28] J. Putaala, O. Nousiainen, M. Komulainen, T. Kangasvieri, H. Jantunen and M. Moilanen, "Influence of Thermal-Cycling-Induced Failures on the RF Performance of Ceramic Antenna Assemblies," IEEE Transactions on Components, Packaging and Manufacturing Technology, vol. 1, pp. 14651472, 2011.

[29] L. Xie, M. Mäntysalo, A. L. Cabezas, Y. Feng, F. Jonsson and L. Zheng, "Electrical performance and reliability evaluation of inkjet-printed $\mathrm{Ag}$ interconnections on paper substrates," Mater. Lett., vol. 88, pp. 68-72, 12/1, 2012.

[30] U. Caglar, K. Kaija and P. Mansikkamäki, "Analysis of mechanical performance of silver inkjet-printed structures," in Proc. 2nd IEEE
International Nanoelectronics Conference (INEC), Shanghai, China, 2008, pp. 851-856.

[31] E. Halonen, V. Pynttäri, J. Lilja, H. Sillanpää, M. Mäntysalo, J. Heikkinen, R. Mäkinen, T. Kaija and P. Salonen, "Environmental protection of inkjet-printed Ag conductors," Microelectron. Eng., vol. 88, pp. 29702976, 2011.

[32] E. Halonen, T. Viiru, K. Östman, A. Cabezas and M. Mäntysalo, "Oven Sintering Process Optimization for Inkjet-Printed Ag Nanoparticle Ink," IEEE T. Compon. Packag. Manuf., vol. 3, pp. 350-356, 2013.

Jussi Putaala received the M.Sc., Lic.Tech., and D.Sc. degrees in electrical engineering from the University of Oulu, Oulu, Finland, in 2005, 2009, and 2015 , respectively.

$\mathrm{He}$ is currently with the Microelectronics Research Unit with the same institute. His current research interests include novel interconnection types and their reliability and prognostic monitoring.

Juha Niittynen received the M.Sc. and D.Sc. degrees in electrical engineering from the Tampere University of Technology (TUT), Tampere, Finland, in 2010 and 2015, respectively.

He is currently with the Meyer Burger B.V., Eindhoven, The Netherlands. His current research interests include characterization and development of inkjet-printed electronics fabrication technology.

Jari Hannu received his M.Sc. and D.Sc. degrees in electrical engineering from the University of Oulu, Oulu, Finland, in 2005 and 2013, respectively. $\mathrm{He}$ is a member of the electrical engineering study program board at the department of electrical engineering with the same institute.

$\mathrm{He}$ is currently a researcher in the Microelectronics Research Unit, University of Oulu, Oulu, Finland. His research interests include board and system level mixed-signal testing of electronics, integrated health and usage monitoring systems for complex systems, and electronics components manufacturing technologies.

Sami Myllymäki received the M.Sc. and D.Sc. degrees in Electrical Engineering from the University of Oulu, Oulu, Finland in 1999 and 2012, respectively. $\mathrm{He}$ has worked in RF engineer, RF specialist, and project manager positions at LK-Products and Nokia corporations, and as a researcher at the University of Tampere, Tampere, Finland.

$\mathrm{He}$ is currently as a researcher in the field of RF engineering and lecturer in basic and advanced courses at the Microelectronics Research Unit, University of Oulu, Oulu, Finland.

Esa Kunnari received the M.Sc. degree at Tampere University of Technology (TUT), Finland, in 2007. His research interests include environmental aspects of printed electronics as well as the development of electronics printing processes. He is currently with the Tampere University of Applied Sciences, Tampere, Finland.

Matti Mäntysalo received his M.Sc. and D.Sc. (Tech) degrees in electrical engineering Tampere University of Technology, Tampere, Finland in 2004 and 2008, respectively. He is an Associate Professor in Electronics materials and manufacturing, Academy research fellow, and has awarded with Adjunct Professor in Digital fabrication. Mäntysalo has led the Printable Electronics Research Group at TUT since 2008. He was a visiting scientist in iPack Vinn Excellence Center, School of information and Communication Technology, KTH Royal Institute of Technology, Stockholm, Sweden, from 2011 to 2012.

His research interests include printed electronics materials, fabrication processes, pre- and post-treatments, quality and performance analyses, reliability and failure analyses, and especially integration of printed electronics with silicon-based technology for healthcare and Internet-ofEverything applications.

Juha Hagberg received the M.Sc., Lic.Tech., and D.Sc. degrees from the University of Oulu, Oulu, Finland in 1987, 1991, and 1993, respectively. He has studied manufacturing technologies for high temperature superconductor thick and thin films and high resolution printing processes for electrical circuitry at the Microelectronics Research Unit and the industry since 1988. 
Docent Hagberg is working as a lecturer at the Microelectronics Research Unit, University of Oulu, Oulu, Finland.

Heli Jantunen received her Dr. Tech. degree in microelectronics from the University of Oulu in 2001 and is working in Microelectronics Research Unit as full professor in Technical Physics. She is also a member of the Research Council for Natural Sciences and Engineering, Academy of Finland, the ESF Standing Committee for Physical and Engineering Sciences (PESC).

Currently she is devoted to her ERC (European Research Council) Advanced Grant project. Her research interests include novel sensors and RF applications by implementation of advanced microwave and functional materials, structures and nanotechnology into multifunctional micromodules and printed electronics devices.

Copyright ( 2016 IEEE. Personal use of this material is permitted. However, permission to use this material for any other purposes must be obtained by sending a request to pubs-permissions@iee.org 